\title{
El proceso salud enfermedad y la transdisciplinariedad
}

\author{
The health-disease process and transdisciplinarity
}

\author{
Lic. Liliana Libreros Piñeros \\ Universidad de Carabobo. Valencia, Venezuela.
}

\begin{abstract}
RESUMEN
La finalidad de este ensayo es reflexionar sobre la naturaleza multidimensional y compleja del proceso salud enfermedad que demanda un abordaje epistémico desde la transdisciplinariedad. Es a partir de las interacciones entre las diferentes disciplinas, que emergen alternativas más completas para solucionar los problemas de salud de la humanidad que rebasan el modelo biomédico. Este modelo ha constituido la base conceptual de la medicina científica moderna, donde el cuerpo humano es fragmentado y analizado desde el punto de vista de sus partes, la enfermedad es el funcionamiento defectuoso de los mecanismos biológicos o químicos y la función de la práctica médica es intervenir física o químicamente para corregir las disfunciones de un mecanismo específico. Se reconocen los innumerables logros de este modelo biomédico desde el siglo XVII hasta el presente, pero es necesario complementar con otros modelos o caminos que permitan comprender la complejidad del ser humano, como un ser que surge de las interacciones entre lo histórico, lo biológico, lo social, lo espiritual y lo cultural. El reto es pues, atreverse a un auténtico diálogo entre saberes para generar cambios en las estructuras políticas, económicas, y culturales de las diferentes sociedades que posibiliten mejorar las condiciones de vida y de salud de las personas.
\end{abstract}

Palabras clave: salud, enfermedad, transdisciplina, complejidad.

\begin{abstract}
The purpose of this paper was to reflect on the multidimensional and complex nature of the health-disease process that demands an epistemic approach from transdisciplinarity. The interactions among the different disciplines give rise to most
\end{abstract}


comprehensive alternatives to respond to human health problems that go beyond the biomedical model. Such a model has been the conceptual basis of the modern scientific medicine in which the human body is fragmented and analyzed from the point of view of its parts; hence, the disease is the malfunctioning of the biological or chemical mechanisms, and the role of the medical practice is to intervene physically or chemically to rectify the dysfunction of a specific mechanism. The numerous accomplishments of this biomedical model since the 17th century up to the present were acknowledged, but it is necessary to supplement it with other models or ways to understand the complexity of the human being, since it emerges from the interactions among the historical, the biological, the social, the spiritual and the cultural elements. Therefore, the challenge isto dare to establish a genuine dialogue among various disciplines to bring out changes in the political, economic and cultural estructures of the different societies toward improving the people's living conditions and health status.

Key words: health, disease, transdisciplinarity, complexity.

\section{PROCESOS HISTÓRI COS}

El derecho a la salud surge con la Revolución Francesa en 1791 y es la Organización Mundial de la Salud (OMS), en el principio $2^{\circ}$ de su Carta Fundacional y en el artículo 25 de la Declaración Universal de los Derechos Humanos (1948) que generaliza ese derecho para todas las personas, sin distinción de raza, religión, ideología política y condición económica o social. ${ }^{1}$ I gualmente en el artículo 84 de la Constitución de la República Bolivariana de Venezuela, se establece el derecho de todos los ciudadanos a la protección de la salud, así como la responsabilidad de los poderes públicos en su organización y tutela. ${ }^{2}$

Es en 1946, cuando la OMS define la salud como "un estado de completo bienestar físico, mental y social y no solo la ausencia de afecciones o enfermedades" (Guardian M. Promoción de la Salud. Discurso de la viceministra de la Salud de Nicaragua en el Día Mundial de la Salud.Managua; 2002). Posteriormente, en 1986 se subscribe la Carta de Ottawa, la cual especifica que la promoción de la salud consiste en proporcionar a los pueblos los medios necesarios para mejorar su salud y ejercer un mayor control sobre la misma; y en 1997 en la Declaración de Yakarta, se reconoce que es solo sobre la base del diálogo entre gobierno y ciudadanos, entre expertos y legos, entre pobres y ricos, que puede pretenderse a tan colosal aspiración de cambio de conducta para el desarrollo; y articular esta conversación universal para el mejoramiento de la salud, que es un delicado compromiso que todos debemos asumir.

A pesar de estos avances en la concepción del proceso salud enfermedad, podemos observar, que aún sigue persistiendo la concepción moderna que visualiza el paradigma orgánico funcionalista del saber médico, que define la estructura corporal como una suma de procesos bioquímicos y fisiológicos. Se trata de un dato objetivamente definido en términos de magnitudes observables y cuantificables y no como un objeto producido en condiciones sociales y del conocimiento humano dadas. ${ }^{3}$ 
Entre los pensadores que influyeron considerablemente en esta medicina moderna, que desde entonces se piensa en términos de Ciencia, Razón y Progreso, tenemos a Comte, inspirado a su vez en otros teóricos predecesores como Kant, Descartes y Bacon, que postulaban una posición mecanicista de la vida, basada en la visión cartesiana y en los principios newtonianos, es decir que tenían una visión reduccionista de los fenómenos; ya que el modelo cartesiano le da importancia al estudio de la estructura y funcionamiento de los fenómenos y descarta las interacciones de los diferentes sistemas con el entorno.

Descartes con la rigurosa separación que hizo entre mente y cuerpo, llevó a los médicos a concentrarse en el cuerpo "máquina" y a olvidar las dimensiones psicológicas, sociales y ambientales del proceso salud enfermedad.

Asimismo, Bacon consolida el carácter experimental del conocimiento científico basado fundamentalmente en los teoremas físicos y matemáticos, se impone entonces, el llamado modelo hipotético deductivo, que es el único lenguaje en las investigaciones científicas, por lo que parece que existía una sola lectura "objetiva" de la realidad.

Son innumerables los logros desde el siglo XVII hasta el presente, que se obtienen bajo esta perspectiva del proceso salud enfermedad, entre ellos, los realizados por Giovanni Borelli, con la explicación de la función muscular, William Harvey, y su explicación de la circulación sanguínea, la invención del microscopio, que genera la investigación en Microbiología con los estudios de Pasteur y Koch y el desarrollo de una nueva ciencia, la Bioquímica; surge así la unicausalidad de la enfermedad, lo cual produce a su vez toda una taxonomía de la enfermedad y una especialización de la medicina.

La aparición de gran cantidad de medicamentos y vacunas para combatir las enfermedades infecciosas y la mejora en las técnicas de cirugía, marcan el inicio del auge de la dependencia de la medicina de la alta tecnología y en su conjunto, favorecen el aumento en la esperanza de vida. En el siglo xx se descubre la cadena de ADN, y se forja así la investigación en Biología Molecular.

En efecto, esta concepción mecanicista, naturalista del proceso salud enfermedad, ha obtenido muchos logros en la mejora de la salud del hombre, pero no es suficiente para interpretar las interacciones entre mente cuerpo ambiente, como sistemas complejos e históricos, además de que dificulta o limita el dialogo entre las diferentes disciplinas, con lo cual se podría comprender mucho mejor los acontecimientos del ser humano en función de todas sus dimensiones biofisiológicas, psicológicas, socioambientales y espirituales.

\section{LA TRANSDI SCI PLI NARI EDAD}

La complejidad de las realidades del mundo actual, exigen superar la parcelación y fragmentación del proceso salud enfermedad, ya que todos los sistemas o estructuras dinámicas que constituyen nuestro mundo: atómicos, moleculares, celulares, biológicos, psicológicos, sociológicos, culturales, entre otros, se caracterizan por sus interconexiones, son todos recíprocamente interdependientes y se rebelan precisamente, porque así reducidos pierden las cualidades emergentes del "todo" y la acción de este sobre cada una de las partes. Esto equivale a decir que debemos pasar de los saberes monodisciplinarios a los multidiscilpinarios, interdisciplinarios y transdisciplinarios. 
El término transdisciplinariedad, que apareció hace tres décadas de manera casi simultánea en los trabajos de diferentes investigadores como Jean Piaget, Edgar Morin, Eric Jantsch y muchos otros, ${ }^{4}$ se ideó en aquel momento para rebasar la pluri y la interdisciplinariedad.

Este enfoque transdisciplinario, se concibe como un conocimiento superior emergente, fruto de un movimiento dialéctico del pensamiento, que permite cruzar los linderos de diferentes áreas del conocimiento disciplinar y crear imágenes de la realidad más completas y más integradas.

Así, el verdadero espíritu de la transdisciplinariedad va más allá de todo lo que prácticamente se está haciendo hasta el presente, su meta o ideal no consiste solo en la unidad del conocimiento, que es considerada como un medio, sino que camina hacia la autotransformación y hacia la creación de un nuevo arte de vivir. Por ello, la actitud transdisciplinaria implica la puesta en práctica de una nueva visión transcultural, transnacional, transpolítica y transreligiosa. Con el dialogo como instrumento operativo se pretende asimilar, o al menos comprender, las perspectivas y el conocimiento de los otros, sus enfoques y sus puntos de vista, y también desarrollar en un esfuerzo conjunto, los métodos, las técnicas y los instrumentos conceptuales que faciliten o permitan la construcción de un nuevo espacio intelectual y de una plataforma mental y vivencial compartida. ${ }^{5}$ (Ugas G. La Complejidad. Un modo de pensar. 2da ed. Venezuela: Taller permanente de estudios epistemológicos en Ciencias Sociales; 2008).

En otras palabras, los postulados o principios básicos de la transdisciplinariedad son: la naturaleza sistémica y la complementariedad. Según Bertalanffy, en un "sistema" se da un "conjunto de unidades interrelacionadas de tal manera que el comportamiento de cada parte depende del estado de todas las otras, pues todas se encuentran en una estructura que las interconecta". Asimismo, Montaigne afirma que la "cualidad más universal es la diversidad" y solo con el "diálogo y con el intercambio con los otros espectadores, especialmente con aquellos ubicados en posiciones contrarias podemos lograr enriquecer y complementar nuestra percepción de la realidad. ${ }^{5}$

En este contexto, la conceptualización del cuerpo humano y el proceso salud enfermedad, debe superar la visión cartesiana que concibe los organismos vivientes como máquinas constituida por diferentes partes, que funcionan según cadenas lineales de causa y efecto, y cuando se estropean es posible identificar una única causa de la avería. Por el contrario, el hombre como organismo viviente funciona como un sistema abierto, y esto significa que debe mantener un intercambio continuo con su entorno para seguir viviendo.

Es decir, que el cuerpo humano funciona como un organismo que procesa fenómenos como la autoorganización, autorrenovación y autotrascendencia. Solo por nombrar algunos ejemplos de estos procesos tenemos, que el páncreas remplaza la mayoría de sus células cada $24 \mathrm{~h}$, la mucosa del estómago cada tres días, los glóbulos blancos de la sangre se renuevan cada 10 días y el $98 \%$ de las proteínas del cerebro lo hacen en menos de un mes. ${ }^{6,7}$

Cabe mencionar el estudio realizado por Mckeown, sobre la evolución de la mortalidad por tuberculosis en Inglaterra y Gales, que solo parece explicarse desde una interpretación sociohistórica y económica política. Las causas de este proceso se deben a una serie de factores como la mejora de la alimentación y su impacto en la resistencia de la población, así como al conjunto de factores socioeconómicos asociados con la revolución industrial, el desarrollo de técnicas de conservación de 
alimentos, la mejora del transporte de mercancías y de las condiciones de las viviendas. ${ }^{6,8}$

De la misma manera es explicable la existencia hoy en día de un mapa planetario de enfermedades caracterizado por un mundo pobre con enfermedades infecciosas endémicas vinculadas a las condiciones de vida deficientes y un mundo rico con enfermedades crónicas y degenerativas relacionadas con el estrés, la alimentación rica en grasas y proteínas, el abuso de drogas, la vida sedentaria y la contaminación ambiental; estas realidades no pueden entenderse desde la doctrina de la etiología específica, sino a partir de la interacción entre mente, cuerpo y entorno, es decir, del papel que juegan los factores biofisiológicos, sociales y culturales en las redes multicausales que producen la enfermedad y la muerte.

En consonancia, el enfoque de la medicina china esta centrada en la interdependencia entre mente, cuerpo y entorno en constante dinamismo. La enfermedad no se concibe como la intrusión de un agente externo, sino como el resultado de varias causas que llevan a la discordia y al desequilibrio. Por otro lado, la naturaleza de todas las cosas, y también del organismo humano, es tal que hay una tendencia natural a recuperar el estado de equilibrio dinámico, por lo tanto, cada persona, según la visión china, es responsable del mantenimiento de su propia salud, y en gran medida también es responsable de su recuperación cuando el organismo entra en un estado de desequilibrio; por lo tanto la función del médico chino, es tratar de restablecer el equilibrio y la armonía, usando varias técnicas terapéuticas destinadas a estimular el organismo del paciente de modo que siga su tendencia natural a retornar a su estado de equilibrio. ${ }^{8}$

También, en Rumania la endocrinóloga Aslan, comenta que hay que valorar mucho la química producida por la "voluntad de vivir". Ella afirma que hay una conexión directa entre una fuerte voluntad de vivir y los balances químicos del cerebro, que una robusta voluntad de vivir produce los impulsos vitales cerebrales que estimulan la glándula pituitaria, la cual dispara, a su vez, una serie de efectos en la glándula pineal $y$ en todo el sistema endocrino. ${ }^{6}$

Simonton, crea una terapia en el tratamiento del cáncer que utiliza el asesoramiento psicológico y la psicoterapia y se puede considerar holística por excelencia. Normalmente esta terapia se realiza en sesiones con grupos de pacientes en las que estos se ayudan y se apoyan mutuamente, está centrada en sus problemas emocionales, sin separarlos de los modelos más generales de sus vidas, y por consiguiente, suele incluir aspectos sociales, culturales, filosóficos y espirituales, y sus variadas interrelaciones. ${ }^{5}$

Como apunta el estudio de Bloom y Monterrossa, que el simple etiquetamiento supone una mayor degradación del nivel general de la salud y la aparición de síntomas depresivos debido a la emocionalidad y sus correlatos psicobiológicos y neuroquímicos. Algo parecido fueron los resultados de la investigación realizada por Berkman y Syme, tras un seguimiento en el tiempo de aproximadamente diez años a un grupo de sujetos. Estos investigadores observan que la falta de red social podía entenderse como un factor de riesgo de mortalidad. ${ }^{8}$

Es decir, que el proceso salud enfermedad, no puede comprenderse en toda su extensión sin tener en cuenta el papel de la cultura y las relaciones sociales. Los síndromes de empacho, susto, pasmo, bilis, ataque de nervios, mal de pelea, mal de ojo, culebrilla, entre otros, se producen exclusivamente en contextos culturales concretos y que, por tanto, no parecen responder a esa lógica biomédica de universalidad de la morbilidad y mortalidad. 
Como dice Morín:

El misterio humano va unido al misterio de la vida y al misterio del cosmos, puesto que llevamos en nosotros la vida y el cosmos. Individuo, sociedad, especie, son de este modo antagonistas al tiempo que complementarios. I mbricados el uno en la otra, no están verdaderamente integrados el uno en la otra; hay una hiancia de muerte entre el individuo efímero y la especie permanente, hay antagonismo del egocentrismo y del sociocentrismo. Cada uno de los términos de esta trinidad es irreductible aunque dependa de los otros. Esto constituye la base de la complejidad humana. ${ }^{9,10}$

Frente a este enfoque de la complejidad del ser humano y de su existencia planetaria, es necesario abordar la salud y la enfermedad desde la perspectiva de redes multicausales en su etiología, donde deben tener cabida las diversas ciencias o todos los conocimientos bien establecidos, ya sea que provengan de la Medicina Social, la Epidemiología Social, la Etnografía, la Psicología, la Antropología Médica, la Física Cuántica y Relativista o las Neurociencias, entre otras.

Pero no como la suma de disciplinas o la hegemonía de una sobre las otras, sino mediante el abordaje epistémico de la transdisciplinariedad, para caminar hacia la integración y conciliación de las diferentes disciplinas y contextos que nos puedan aclarar y aproximar a las diferentes realidades complejas y dar una respuesta más completa y más integrada a los acontecimientos de la salud y la enfermedad del hombre, mediante nuevos modos de producción de conocimiento y aprovechar los logros obtenidos en la modernidad como el uso de la tecnología avanzada pero sin detrimento del relato del paciente, de su cultura, sus representaciones, sus condiciones sociales y materiales de existencia, su mundo local, sus emociones, es decir, transcender a la observación e interpretación, no de la enfermedad sino del ser humano en cada una de sus dimensiones, en dinámica interacción entre éstas, el entorno y el cosmos.

\section{DESAFÍ OS}

Este cambio de paradigma no es tarea fácil, pero al parecer y muy lentamente hemos comenzado el camino, ya es un logro reconocer las debilidades del paradigma moderno, de las disciplinas atrincheradas con sus verdades absolutas e irrefutables y atrevernos en la incertidumbre de un nuevo paradigma, la transdiciplina, al abordaje del proceso salud enfermedad, para lo cual es necesario un auténtico diálogo entre saberes así como un cambio en las estructuras políticas, económicas y culturales de las diferentes sociedades para lograr soluciones a los graves problemas del hombre y del planeta.

\section{REFERENCI AS BI BLI OGRÁFICAS}

1. Delgado M, Llorca J. Concepto de salud. El continúo salud-enfermedad. Historia natural de la enfermedad. Determinantes de la salud. En: Hernández I, Gil A, Delgado M, Bolumar F, editores. Manual de Epidemiología y Salud Pública. Madrid: Médica Panamericana, s.a; 2005. p. 3-6. 
2. Constitución Bolivariana de la República de Venezuela, gaceta $N^{\circ}$. 36.860. Jueves 30 de Diciembre de 1999.

3. Rodriguez F. Sujeto, Episteme y posmodernidad. Venezuela: Fundacite Guayana; 2006.

4. Nicolescu B. Transdisciplinariedad. Manifiesto. México: Multiversidad mundo real "Edgar Morín, A.C"; 1996. (Traducción de Mercedes Vallejo Gómez).

5. Martínez M. Epistemología y Metodología Cualitativa en las Ciencias Sociales. México, D.F.: Trillas, s.a. de C.V; 2008.

6. Martínez M. El Paradigma Emergente. Hacia una nueva teoría de la racionalidad científica. 2da ed. México, D. F.: Trillas, s.a de C.V; 2007.

7. Martinez M. La Nueva Ciencia. Su desafío, lógica y método. México, D. F.: Trillas, S.a de C.V; 1999.

8. Martínez A. Antropología Médica. Teorías sobre la cultura, el poder y la enfermedad. España: Anthropos; 2008.

9. Morín E. La cabeza bien puesta. Repensar la reforma. Reformar el Pensamiento. Buenos Aires: Nueva visión; 2007.

10. El Método. La humanidad de la humanidad. La identidad humana. España: Editions du Seuil; 2003.

Recibido: 23 de septiembre de 2011.

Aprobado: 30 de abril de 2012.

Liliana Libreros. Departamento de Salud Pública de la Escuela de Salud Pública y Desarrollo Social de la Universidad de Carabobo. Valencia, Venezuela. Telf:

04144252231-0241-8435420. Correo electrónico: lilianalibreros05@yahoo.es 